\title{
Desenvolvimento, Psicopatologia e Apego: Estudo Exploratório com Crianças Institucionalizadas e suas Cuidadoras
}

\author{
Development, Psychopathology and Attachment: An Exploratory Study with Institu- \\ tionalized Children and their Caregivers
}

\author{
Mariana Pereira*, ${ }^{*}$, Isabel Soares ${ }^{a}$, Pedro Dias $^{b}$, Joana Silva $^{a}$, Sofia Marques $^{a}, \&$ Joana Baptista $^{c}$ \\ ${ }^{a}$ Universidade do Minho, ${ }^{b}$ Universidade Católica Portuguesa \& ${ }^{c}$ Universidade do Porto
}

\begin{abstract}
Resumo
O presente estudo exploratório examinou o desenvolvimento mental e a qualidade do funcionamento sócio-emocional de 16 crianças entre os 3 e os 6 anos, institucionalizadas em Centros de Acolhimento Temporário, relacionando-os com a qualidade das narrativas sobre o apego das suas cuidadoras. Foram utilizadas a Escala de Desenvolvimento Mental de Griffiths, o Questionário de Comportamentos, as Narrativas sobre o Apego e o Attachment Q-Sort. Os resultados revelaram que o nível de desenvolvimento das crianças foi inferior aos valores normativos. Além disso, os valores apresentados ao nível da psicopatologia, em termos de sintomas de internalização e de externalização, aproximaram-se dos valores clínicos. Não obstante e, em contraste com o esperado, a maioria das crianças apresentou valores próximos da segurança (base segura), os quais não estão associados com a qualidade das narrativas sobre o apego das suas cuidadoras. Os resultados são discutidos com base no impacto da privação em meio institucional para o desenvolvimento na infância.

Palavras-chave: Infância; Institucionalização; Apego; Desenvolvimento; Psicopatologia.

Abstract

This exploratory study examined mental development and the quality of socio-emotional functioning of 16 institutionalized children, aged from 3 to 6 years old, relating them with the quality of their caregivers' attachment narratives. Griffiths Developmental Scales, Child Behavior Checklist, Attachment Script Representation and Attachment Q-Sort were used in the psychological assessment. Results showed that chidren's developmental level was placed below normative data. Furthermore, data regarding externalizing and internalizing psychopathology symptoms were close to the clinical population. Nevertheless, and in contrast to our hypothesis, most children presented almost mormative attachments scores (secure base), but this was not related to the quality of attachement narratives presented by their caregivers. The implications of these findings are discussed in light of the impact of institutional deprivation in child development.

Keywords: Infancy; Institutionalization; Attachment; Development; Psychopathology.
\end{abstract}

A institucionalização tem emergido como uma medida alternativa de prestação de cuidados em percursos de vida marcados por diversas situações adversas associadas a orfandade, mau-trato, negligência e/ou abandono. A proliferação de estudos focalizados nesta temática permitiu acumular evidência empírica (in)directa acerca do impacto do acolhimento no desenvolvimento da infância, documentando que a experiência disruptiva nos cuidados e a vivência institucional se relacionam com resultados desenvolvimentais negativos posteriores (Nelson et al., 2007; O'Connor, Bredenkamp, Rutter \& The English and Romanian Adoptees [ERA] Study Team, 1999; Provence \& Lipton, 1962; Zeanah, Smyke, Koga,

* Endereço para correspondência: Universidade do Minho, Instituto de Educação, Campus de Gualtar, Braga, Portugal, 4710-057.E-mail: marianamferreira@live.com.pt
Carlson, \& The Bucharest Early Intervention Project Core Group [BEIP], 2005).

Diversas investigações têm revelado uma maior prevalência de problemas comportamentais e sócio-emocionais graves ao nível das relações com os pares e com os adultos (Cicchetti \& Tucker, 1994; Fischer, Ames, Chisholm \& Savoie, 1997; Tizard \& Rees, 1975). Neste âmbito, a integração precoce em meio institucional tem sido associada ao apego inseguro (Hortacsu \& Cesur, 1993; Marcovitch et al., 1997), mas também a perturbações graves do apego, designadamente na capacidade de organização de uma relação com as figuras prestadoras de cuidados na instituição (Zeanah et al., 2005) e no desenvolvimento do apego atípico (Vondra \& Barnett, 1999). Diferentes estudos têm suportado um padrão de comportamento muito perturbado caracterizado por sociabilidade indiscriminada face a figuras adultas estranhas 
e por interacções afectuosas mas superficiais (Chisholm, 1998; O’Connor, Rutter, Becket, Keaveney, Kreppner \& ERA Study Team, 2000; Tizard \& Rees, 1975).

Características do contexto institucional, como maior atenção à prestação dos cuidados físicos e menor atenção ao estabelecimento de relações e cuidados individualizados, não permitem responder de modo adequado às necessidades específicas das crianças (Frank, Klass, Earls \& Eisenberg, 1996). Uma escassa estimulação motora, sensorial e cognitiva tem sido relacionada com défices desenvolvimentais significativos, tais como: atraso no desenvolvimento da linguagem e de funções cognitivas, no crescimento físico e psicomotor (Nelson et al., 2007; Provence \& Lipton 1962; Van IJzendoorn \& Juffer, 2006).

Além disso, alterações no desenvolvimento e no funcionamento da actividade neuroendócrina, designadamente uma produção de cortisol diurna elevada, têm também vindo a ser entendidas como resultados da privação de cuidados parentais (Gunnar, Plorison, Chisholm, \& Schuder, 2001).

As associações encontradas nestes trabalhos não são, todavia, lineares. Se, por um lado, a maioria destes estudos recorre a dados retrospectivos e enfatiza factores de natureza individual, concedendo pouca importância ao contexto relacional e institucional, por outro, a plasticidade do organismo humano e a diferenciação individual precipitam trajectórias desenvolvimentais distintas. A presença (ou ausência) de recursos pessoais e sociais (McCall \& Groark, 2000), a qualidade dos cuidados recebidos, o tempo de institucionalização e a idade de entrada na instituição (O'Connor et al., 2000; Zeanah et al., 2005) são alguns dos factores assinalados como mediadores dos efeitos da institucionalização. No entanto, o suporte empírico de que o contexto institucional não favorece o desenvolvimento adequado da criança é incontestável. Efectivamente, vários estudos têm evidenciado efeitos nocivos nos domínios físico, neurobiológico, cognitivo, comportamental, sócio-emocional e interpessoal (Johnson, Brown, \& Hamilton-Giachritsis, 2006; Nelson et al., 2007; Soares, 2007).

Apesar de uma significativa variabilidade intra-grupo e de não serem ainda conhecidos os processos através dos quais ocorre este impacto negativo, a má qualidade da prestação de cuidados (Smyke et al., 2007) e, em muitos casos, a ausência do estabelecimento de uma relação de apego com um prestador de cuidados (Bowlby, 1973; Johnson et al., 2006; O'Connor et al., 1999) parecem estar associadas a resultados desenvolvimentais futuros perniciosos, na medida em que comprometem a vivência de experiências de afecto positivo e de regulação emocional adequadas e a progressiva aquisição de conhecimentos e expectativas acerca: (a) da figura de apego como disponível, responsiva e apoiante à exploração do meio; (b) do self como competente, valorizado e capaz de influenciar os outros; (c) do mundo em geral (Bretherton \& Munholland, 1999).
Nelson et al. (2007) ressaltam que a questão crítica da plasticidade cerebral reside na relevância dos períodos sensíveis do desenvolvimento, a partir dos quais a recuperação pode estar seriamente comprometida. Deste modo, a intervenção precoce junto de crianças institucionalizadas e o retorno a um contexto familiar assumem distinta saliência. Nesta linha, o estudo de Dozier, Stovall, Albus e Bates (2001) revelou que crianças integradas em famílias de acolhimento até ao primeiro ano de vida são capazes de orientar e organizar o seu comportamento de apego para a nova figura prestadora de cuidados. Por seu lado, a meta-análise conduzida por Van IJzendoorn e Juffer (2006) evidencia que, quando a adopção acontece após os 12 meses de idade, isto é, após o período em que a criança aprende a confiar num adulto sensível, responsivo e disponível que opera como base segura - o risco da criança desenvolver dificuldades na organização de uma relação de apego vai aumentando significativamente. Assim, apesar da colocação em ambiente institucional, é possível que a criança venha a estabelecer uma relação de apego organizada com uma figura parental desde que, em tempo oportuno, lhe seja assegurada uma elevada qualidade na prestação de cuidados, e uma figura sensível e responsiva capaz de a ajudar a ultrapassar as suas limitações e dificuldades (Dozier et al., 2001).

Considerando que o impacto desfavorável da institucionalização no desenvolvimento das crianças se deve, para além de outros factores, à organização do contexto institucional, o nosso estudo procura contribuir para o conhecimento da vivência institucional da infância, em Portugal, onde são muito escassos os estudos científicos nesta área. Neste sentido, o presente trabalho tem como principal objectivo examinar o desenvolvimento e o funcionamento sócio-emocional de crianças em idade pré-escolar acolhidas em Centros de Acolhimento Temporário (CATs) das regiões Norte e Centro do país, bem como a qualidade dos cuidados prestados pelos seus cuidadores de referência. Os objectivos específicos deste trabalho são: (a) avaliar o desenvolvimento mental da criança e presença de sintomatologia psicopatológica; (b) identificar a representação de apego do prestador de cuidados de referência e a qualidade da relação de apego da criança com o seu cuidador; (c) analisar as relações entre qualidade do apego, representação de apego do cuidador, desenvolvimento e sintomatologia psicopatológica.

\section{Método}

\section{Participantes}

Neste estudo participaram 16 crianças residentes em três CATs da região Centro $(n=11)$ e três CATs da região Norte $(n=5)$ do país, com idades compreendias entre os 3 e os 6 anos $(M=4,06$ anos, $D P=0,96)$, sendo 10 do sexo feminino e 6 do sexo masculino. 
O tempo médio de institucionalização foi de 15,69 meses $(D P=12,53)$, com um tempo de permanência mínimo de 6 meses $(n=2)$ e máximo de 47 meses $(n=1)$. A negligência destaca-se como o principal motivo que conduziu à institucionalização, sendo seguida, por ordem decrescente, da desorganização familiar, do abuso sexual/abandono e da toxicodependência/alcoolismo dos progenitores. Uma pequena maioria $(n=9)$ teve a sua primeira medida de protecção na colocação em CAT, sendo que 7 crianças estiveram colocadas noutra instituição ou tiveram outra medida de acolhimento antes de darem entrada na actual instituição.

As prestadoras de cuidados de referência $(n=12$; cuidadoras de quatro crianças eram também figura de referência de outra criança) tinham idades compreendidas entre os 22 e os 37 anos $(M=27,19, D P=4,65)$. O tempo médio do exercício das suas funções era de 3,5 anos $(D P=3,08)$, sendo o limite inferior de 1 ano e o limite superior de 11 anos. As suas habilitações literárias variaram entre a frequência do Ensino Pós-graduado $(n=2)$, Ensino Superior ( $n=5)$, Bacharelato $(n=1)-$ designadamente nas áreas da Educação e das Ciências Sociais/Humanas -, Ensino Secundário $(n=3)$ e $9^{\circ}$ ano $(n=5)$.

As directoras dos CATs $(n=6)$ tinham idades compreendidas entre os 31 e os 47 anos $(M=41, D P=5,83)$. O exercício das suas funções variava entre 9,67 anos $(D P=5,5)$, sendo o limite inferior de 4 anos e o limite superior de 17 anos. A distribuição em função das suas habilitações literárias indicou que todas frequentaram o Ensino Superior, especificamente na área das Ciências Sociais e Humanas, além de que três prosseguiram com estudos pós-graduados.

\section{Instrumentos}

Medidas Relativas à Criança. Escala de Desenvolvimento Mental de Ruth Griffiths (1970) - constituída por seis sub-escalas (locomoção, pessoal-social, audição e linguagem, coordenação óculo-manual, realização, raciocínio prático), os somatórios dos itens bem sucedidos em cada uma delas foram convertidos num quociente de desenvolvimento padronizado, ponderado de acordo com a amostra normativa correspondente à respectiva faixa etária da criança.

Para a administração desta escala, utilizou-se a versão de investigação de Castro e Gomes (1996), que contém as instruções para cada item e sua ordem de apresentação. Cada uma das sub-escalas foi administrada individualmente, tendo sido remetida para o final do procedimento a sub-escala locomotora, dado poder sobrestimar o nível de actividade da criança, condicionando o desempenho ao longo da prova (Griffiths, 1970).

Questionário de Comportamentos da Criança 11/2-5 e 6-18 ([CBCL 11/2-5], Achenbach \& Rescorla 2000; [CBCL 6-18], Achenbach 2001, versões portuguesas de Gonçalves, Dias, \& Machado, 2007a, 2007b) - compostas por
100 e 113 itens (respectivamente) que descrevem comportamentos problemáticos da criança (ex. "É cruel com os animais", "Não quer dormir sozinho", "É pouco activo, vagoroso, tem falta de energia", "Não parece sentir-se culpado depois de se ter comportado mal", "Isola-se, não se envolve com os outros" etc.), cada um deles foi pontuado pelas cuidadoras de referência das crianças segundo uma escala de 3 pontos ( 0 = não verdadeira, $1=$ algumas vezes verdadeira, $2=$ muito verdadeira). $\mathrm{O}$ somatório global dos itens permitiu obter uma pontuação total, que fornece um indicador geral de sintomatologia psicopatológica, enquanto que uma análise factorial de segunda ordem facultou a extracção de dois factores correspondentes às escalas de internalização (síndromas orientados para o self, como por ex. depressão) e de externalização (síndromas orientados para a relação do self com os outros, como por ex. agressão) (Achenbach \& Rescorla 2000).

Para este estudo, dado o seu preenchimento ter sido conduzido pela prestadora de cuidados de referência da criança, foram introduzidas ligeiras adaptações aos itens deste instrumento. Relativamente à CBCL 1 1/2-5, foi apenas substituída a palavra pais do item 37 que passou a designar-se por: "Fica muito aflito/a quando é separado/a de si”. Quanto à CBCL 6-18, foi retirada a palavra pais do item 2 que passou a designar-se por: "Consome bebidas alcoólicas sem consentimento" e foi acrescentada a palavra casa ao item 21 que passou a designar-se por:" Destrói coisas da sua casa ou de outras crianças".

Medidas Relativas à Cuidadora. Narrativas sobre o Apego (Waters \& Rodrigues-Doolabh, 2004, adaptadas para Portugal por Veríssimo \& Santos, 1999) - construídas a partir de seis cartões, compostos por um conjunto de palavras que estimulam conteúdos de apego relevantes referentes à interacção mãe-criança (" $A M a$ nhã do Bebê" [A], "No Consultório do Médico" [B]) e à interacção entre adultos ("O Acampamento da Joana e do Pedro" [C], "O Acidente" [D]) ou que remetem para cenários neutros ("O Passeio no Parque" [E] e "Uma Tarde nas Compras" [F]).

Os blocos de três histórias alusivas às interacções mãecriança e entre adultos foram apresentados numa ordem de seis sequências diferentes, de modo a que a sua distribuição fosse equilibrada.

Um sistema de codificação assente no pressuposto de que um script de base segura preconiza um fenómeno segundo o qual a figura de apego, sensível ao estado emocional do interveniente principal, auxilia a personagem a lidar com o obstáculo/conflito e a retornar à normalidade da situação, permitiu qualificar a representação de apego da prestadora de cuidados. Segundo uma escala de sete pontos, as narrativas de pontuações mais elevadas (6 e 7) correspondem a histórias de conteúdo extenso de base segura e de forte organização interpessoal, enquanto que narrativas de pontuações mais baixas (1 e 
2) expressam um reduzido conteúdo de base segura, com teores incoerentes e/ou bizarros.

Para esta investigação, quatro juízes foram treinados para o efeito por uma formadora de referência a nível nacional e cotaram em grupos de dois, separadamente, os quatro scripts que remetem para conteúdos de apego relevantes (A, B, C e D). Após a transcrição e cotação das histórias relatadas pelas cuidadoras procedeu-se a uma análise correlacional entre os juízes e verificou-se que nenhuma das comparações diferiu em mais de 1 ponto.

Medidas Relativas à Interacção Criança-Cuidadora. Attachment Behavior Q-Sort: versão 3.0 ([AQS], E. Waters, 1995, adaptado para Portugal por Veríssimo \& Santos, 1999) - integra diversos comportamentos relevantes em torno da segurança do apego. No entanto, é a sistematização destes comportamentos no perfil $Q$-Sort que permite qualificar a relação de apego da criança e da interacção criança-cuidadora (Veríssimo \& Salvaterra, 2006a, 2006b).

Partindo da observação da criança em interacção com a sua prestadora de cuidados de referência num ambiente ecologicamente válido e por um período de tempo não inferior a duas horas, os itens que compõem o AQS foram repartidos ao longo de uma escala de 9 níveis distribuídos entre o "extremamente típico" e o "extremamente atípico". Assim, tendo em consideração o grau de relevância para a criança, as afirmações que melhor a caracterizaram foram colocadas na categoria dos comportamentos "típicos" e observados (9-7). As afirmações que nem foram características, nem incaracterísticas e/ ou não observadas, foram colocadas nos comportamentos que "não se aplicam" (6-4). As afirmações que menos caracterizaram a criança, ou que não eram condizentes com o comportamento observado, foram colocadas na categoria dos comportamentos "atípicos" (3-1).

Quatro observadores foram treinados para o efeito por uma formadora de referência a nível nacional e distribuíram em grupos de dois, separadamente, os itens por 9 categorias de comportamento de um modo quasi-normal. $\mathrm{O}$ acordo inter-juízes foi avaliado utilizando o método de bipartição (fórmula de Spearman-Brown), não se tendo registado níveis de acordo insuficientes (Spearman-Brown $<0,65)$. O grau de acordo médio entre os observadores foi de 0,81 (limite mínimo de 0,75 e limite máximo de 0,96 ). O $Q$-Sort de cada criança foi obtido através da média dos dois Sorts. A pontuação média dos dois observadores foi, em seguida, correlacionada com os valores critério de segurança (despreocupação e confiança na disponibilidade da figura de apego) e dependência (procura de contacto e interacção com o prestador de cuidados, normativas na primeira infância) definidos por um conjunto especialistas da área do apego e relativos a uma criança ideal.

Considerando a observação do construto de base segura em visitas domiciliárias, Posada, Waters, Crowell e Lay (1995) desenvolveram quatro escalas, constituídas por itens do AQS, fundamentais para a compreensão deste fenómeno: (a) Interacção suave (harmonia existente entre a criança e a mãe); (b) Proximidade (aproximação/ afastamento à mãe quando perturbada/a necessitar de ajuda e/ou ao saber a sua localização); (c) Contacto físico (prazer no estabelecimento de contacto físico com a mãe e no conforto proporcionado por esta); (d) Interacção com outros adultos (prontidão da criança para interagir com adultos) (Posada, 2006).

Para este estudo empírico foram introduzidas ligeiras adaptações aos itens deste instrumento, tendo-se substituído o item 35 que passou a designar-se por: "A criança é independente de si. Prefere brincar sozinha, deixa-a facilmente quando quer brincar". Foi também alterado o item 26 que passou a designar-se por: "A criança chora quando a deixa em casa com outro cuidador ou outra pessoa".

\section{Procedimento}

A coleta de dados teve início após a obtenção do consentimento informado junto do representante legal responsável pela criança (directoras dos CATs) e decorreu ao longo de três visitas às instituições. Num primeiro contacto, procurou-se obter informação sociodemográfica e descritiva relativa à criança com o auxílio de um elemento da equipe técnica (e.g. directora, psicóloga ou assistente social). Foram excluídas crianças com perturbações graves de desenvolvimento, crianças de 6 anos que frequentassem o primeiro ano do primeiro ciclo do Ensino Básico e crianças institucionalizadas por um período inferior a 6 meses.

O segundo momento de avaliação teve como foco inicial a avaliação do desenvolvimento da criança através da Escala de Desenvolvimento Mental de Ruth Griffiths (1970) e o estabelecimento do primeiro contacto com as prestadoras de cuidados de referência de cada criança. Dada a constatação de que os CATs não dispunham de técnicas identificadas como figuras de apego para cada criança, as cuidadoras que participaram neste estudo foram identificadas com base nos seguintes critérios: (a) passar mais tempo com a criança (e.g. fins-de-semana, épocas festivas) e/ou (b) ser mais responsável pela criança. A não aplicabilidade dos critérios anteriores para duas crianças, levou a que as suas cuidadoras tivessem sido identificadas com base no critério conhecer melhor a criança. Em seguida, procedeu-se à execução da CBCL, preenchida pelas cuidadoras.

Por último, o terceiro contacto com a instituição destinou-se à observação da interacção entre a criança e a sua cuidadora, seguindo-se-lhe a administração das Narrativas sobre o Apego. O AQS, gravado em vídeo durante cerca de duas horas e meia a três horas, foi conduzido por um observador previamente treinado e familiarizado com o instrumento. Procurou criar-se uma atmosfera informal de modo a que a interacção criança-cuidadora decorresse em ambiente natural, sem se interferir ou perturbar as interacções. Contudo, quando solicitado, o observador treinado para o efeito conversou informal- 
mente com a prestadora de cuidados e participou nas brincadeiras das crianças. Sempre que oportuno, questionou a cuidadora acerca de itens de difícil visualização (e.g. item 62. "Quando a criança está bem disposta, é provável que continue todo o dia") e/ou itens que não foram possíveis de serem observados ao longo da visita (e.g. item 47. "Enquanto brinca, a criança aceita e aprecia sons barulhentos ou ser balanceada, se lhe sorrir e mostrar que é divertido"). Quando as observações terminavam, era pedido à prestadora de cuidados que acompanhasse o investigador a uma outra divisão da instituição para que procedesse à tarefa das Narrativas sobre o Apego. As histórias foram gravadas em áudio e decorreram, aproximadamente, ao longo de 15 a 20 minutos. A posteriori, foram realizadas as descrições do $Q$-Sort da criança e as cotações das transcrições das Narrativas sobre o Apego por quatro observadores treinados para o efeito. É importante referir que o grupo de cotadores do AQS divergiu do das Narrativas sobre o Apego.

\section{Resultados}

\section{Desenvolvimento Mental}

O nível global de desenvolvimento dos participantes situou-se, em média, nos 81,38 ( $D P=5,79)$, ou seja, abaixo dos dados normativos apresentados por Griffiths (1970) $(M=100, D P=15)$. Os quocientes globais de desenvolvimento mental encontrados variaram entre 70,29 e 93,44 , tendo-se verificado que mais de metade dos sujeitos $(n=11)$ revelou valores inferiores a 85 .

\section{Psicopatologia}

Esta análise excluiu uma criança com 6 anos a quem foi administrada a CBCL 6-18, sendo que às restantes $(n=15)$ foi administrada a CBCL $1 \frac{112-5}{2}$. A médias das pontuações obtidas na escala de internalização foi de 15,53 ( $D P=7,52)$, sendo o limite mínimo 6 e o limite máximo 33. A média das pontuações registradas na escala de externalização foi de 17,27 $(D P=6,69)$, sendo o limite mínimo 1 e o limite máximo 29. A média das pontuações obtidas no indicador geral de sintomatologia psicopatológica foi de 47,93 ( $D P=17,85)$, sendo o limite mínimo de 12 e o limite máximo de 83 .

Em termos gerais, e tendo por referência os valores revelados nas escalas de internalização e de externalização por Achenbach e Rescorla (2000) para a população normativa (respectivamente $M=8,7 ; D P=6,3$ e $M=13,1$; $D P=7,8$ ), constatou-se que os resultados apresentados em ambas as escalas se aproximaram dos valores encontrados por estes autores para a população clínica (respectivamente $M=17,5 ; D P=10,2$ e $M=19,0 ; D P=11,1)$. Não obstante, o indicador geral de psicopatologia aproximou-se mais do valor normativo $(M=33,4 ; D P=18,8)$ do que do valor clínico $(M=58,8 ; D P=26,5)$.

\section{Narrativas sobre o Apego das Cuidadoras}

A média das pontuações verificadas nas histórias mãe-criança foi de $3,18(D P=0,76)$, sendo os seus limites mínimo de 2 e máximo de 5,25. A média das pontuações observadas nas histórias adulto-adulto foi de 3,74 $(D P=1,02)$, sendo os seus limites mínimo de 2,38 e máximo de 5,5. A média das pontuações obtidas no valor compósito de apego foi de 3,46 ( $D P=0,81)$, sendo os seus limites mínimo de 2,31 e máximo de 5,13. Registou-se portanto, que as narrativas produzidas evidenciaram scores díspares de um script de base segura.

Procurou-se avaliar a relação entre a idade e o nível de escolaridade das cuidadoras e os resultados dos seus scripts. A associação entre a idade das cuidadoras e os resultados das narrativas não se revelou significativa. Todavia, como se pode observar na Tabela 1, com base no coeficiente de correlação de Spearman as relações entre as habilitações literárias e os resultados das narrativas foram significativas. As cuidadoras com maior nível de escolaridade apresentaram scripts de base segura.

Tabela 1

Relação entre Nível de Escolaridade e Narrativas das Cuidadoras

Narrativas Nível de escolaridade

\begin{tabular}{ll}
\hline Histórias mãe-criança & $0,76 * *$ \\
Histórias adulto-adulto & $0,71 *$ \\
Valor compósito de apego & $0,80 * *$ \\
\hline
\end{tabular}

Nota. $* p<0,05 ; * * p<0,01$.

\section{Qualidade da Interacção Criança-cuidadora}

Tendo por referência os critérios de segurança e dependência relativos a uma criança ideal, definidos por um conjunto de peritos, estes dados foram correlacionados com a informação recolhida através do perfil $Q$ Sort, para se alcançarem resultados em ambos os construtos. Deste modo, após correlação com os critérios ideais de segurança e dependência, os resultados observados relativos à segurança $(M=0,34, D P=0,22)$ e à dependência $(M=-0,07, D P=0,23)$, demonstraram que a maioria das crianças apresentou valores de segurança próximos dos encontrados por Park e Waters (1989) ( $M=$ de 0,38 a 0,41$)$ e por Veríssimo e Salvaterra (2006a) $(M=0,39 ; D P=0,16)$. Os valores de segurança encontrados variaram entre $-0,20$ e 0,64 , enquanto que, por sua vez, os valores de dependência variaram entre $-0,56$ e 0,32 . Os resultados exibem apenas um valor de segurança negativo $(n=1)$, em contraste com onze valores de dependência negativos $(n=11)$.

No sentido de analisar com mais detalhe a relação criança-cuidadora, foram utilizadas as escalas de Posada et al. (1995), que a especificam em quatro dimensões, cujos resultados são apresentados na Tabela 2 . 
Pereira, M., Soares, I., Dias, P., Silva, J., Marques, S. \& Baptista, J. (2010). Desenvolvimento, Psicopatologia e Apego: Estudo Exploratório com Crianças Institucionalizadas e suas Cuidadoras.

Tabela 2

Qualidade da Interacção Criança-Cuidadora

\begin{tabular}{lc}
\hline Escalas de Posada et al. (1995) & M (DP) \\
\hline Interacção suave & $4,87(0,30)$ \\
Contacto físico & $5,38(0,60)$ \\
Proximidade & $4,96(0,69)$ \\
Interacção com outros adultos & $5,27(0,96)$ \\
\hline
\end{tabular}

Os valores obtidos nas escalas contacto físico e interacção com outros adultos aproximaram-se dos dados encontrados pela investigação para a população normativa. Em contrapartida, as escalas interacção suave e proximidade revelaram valores ligeiramente mais baixos (Posada, 2006; Veríssimo \& Salvaterra, 2006b).

Procedeu-se à análise da relação entre a idade e os critérios de segurança e dependência, tendo-se observado uma correlação negativa marginalmente significativa entre esta variável e a dependência $(r h o=-0,46 ; p=0,08)$ com base no coeficiente de correlação de Spearman. À medida que a idade da criança aumenta, a sua dependência para com a cuidadora diminui. Não se verificou, todavia, qualquer tipo de associação entre a segurança e a idade $(r h o=0,06 ; p=0,84)$ (Tabela 3).

Procurou-se ainda avaliar a relação entre a idade e as escalas de Posada et al. (1995), tendo-se constatado uma correlação marginalmente significativa entre esta variável e a escala interacção com outros adultos $(r h o=0,43$; $p=0,10)$ com base no coeficiente de correlação de Spearman (Tabela 3). Crianças de maior idade manifestam mais comportamentos de interacção com outros adultos.
Tabela 3

Relações entre a Idade das Crianças e Qualidade da Interacção Criança-Cuidadora

\begin{tabular}{lc}
\hline AQS & Idade \\
\hline Dependência & $-0,46 \dagger$ \\
Segurança & 0,06 \\
Interacção suave & $-0,28$ \\
Contacto físico & $-0,24$ \\
Proximidade & $-0,20$ \\
Interacções outros adultos & $0,43 \dagger$
\end{tabular}

Nota. $\dagger p<0,10$.

Qualidade da Interacção Criança-cuidadora e Narrativas sobre o Apego da Cuidadora

A análise das relações entre o valor critério de segurança e dependência e o valor compósito das narrativas (mãe-criança, adulto-adulto e valor compósito total) não revelou qualquer associação significativa.

No sentido de se compreenderem melhor os resultados, conforme o indicado na Tabela 4, correlacionaram-se os scripts maternos com as escalas de Posada et al. (1995) com base no coeficiente de correlação de Spearman, tendo sido obtido um resultado marginalmente significativo entre as histórias mãe-criança e a escala proximidade $(r h o=0,47 ; p=0,07)$. As histórias mãe-criança que remetem para conteúdos de proximidade relacionam-se com os níveis de proximidade estabelecidos entre a criança e a sua cuidadora. Verificaram-se também correlações negativas estatisticamente significativas entre as histórias mãe-criança e a escala contacto físico e entre o valor compósito de apego e a escala contacto físico. À medida que aumenta o conteúdo de contacto físico ex-

Tabela 4

Relações entre as Narrativas da Cuidadora e a Qualidade da Interacção Criança-Cuidadora

\begin{tabular}{lcccc} 
Narrativas & Interacção Suave & Contacto Físico & Proximidade & Interacções outros adultos \\
\hline Histórias mãe-criança & $-0,27$ & $-0,65^{* *}$ & $0,47 \dagger$ & $-0,49$ \\
Histórias adulto-adulto & $-0,25$ & $-0,36$ & 0,29 & 0,00 \\
Valor compósito de apego & $-0,26$ & $-0,51^{*}$ & 0,38 & $-0,02$ \\
\hline
\end{tabular}

${ }^{*}$ Nota. ${ }^{*} p<0,01 ; * p<0,05 ; \dagger p<0,10$.

Tabela 5

Relação entre os Níveis de Desenvolvimento Mental da Criança e a Qualidade da Interacção Criança-Cuidadora

\begin{tabular}{lcccc}
\hline Desenvolvimento mental & Interacção suave & Contacto físico & Proximidade & Interacção outros adultos \\
\hline Locomotor & $-0,06$ & $-0,14$ & $-0,03$ & 0,21 \\
Pessoal e social & 0,20 & 0,29 & 0,09 & 0,09 \\
Audição e linguagem & 0,26 & $-0,04$ & 0,15 & 0,14 \\
Coordenação óculo-manual & 0,01 & 0,10 & 0,10 & 0,08 \\
Realização & 0,30 & 0,29 & 0,29 & $-0,20$ \\
Raciocínio prático & 0,18 & $-0,22$ & $0,45 \dagger$ & $-0,12$
\end{tabular}

Nota. $\dagger p<0,10$. 
presso nas histórias mãe-criança (e no valor compósito de apego), diminui o contacto físico estabelecido entre a criança e a sua cuidadora.

\section{Qualidade da Interacção Criança-cuidadora e Desenvolvimento Mental da Criança}

As relações encontradas entre os níveis de desenvolvimento mental da criança e as características da interacção com a cuidadora não foram estatisticamente significativas, tendo sido apenas encontrada uma relação marginalmente significativa, com base no coeficiente de correlação de Spearman entre o raciocínio prático e a escala proximidade $(r h o=0,45 ; p=0,08)$, como se observa na Tabela 5. Crianças com melhor raciocínio prático exibem mais comportamentos de aproximação à cuidadora.

\section{Qualidade da Interacção Criança-Cuidadora e Psicopatologia}

Procedeu-se à análise da associação entre os sintomas psicopatológicos e a qualidade do apego da criança, que não revelou correlações estatisticamente significativas (Tabela 6).

\section{Tabela 6}

Psicopatologia e Qualidade da Interacção CriançaCuidadora

\begin{tabular}{lcc}
\hline Psicopatologia & Segurança & Dependência \\
\hline Internalização & $-0,12$ & 0,42 \\
Externalização & $-0,13$ & 0,40 \\
Pontuação total & 0,20 & 0,24 \\
\hline
\end{tabular}

\section{Discussão}

Os resultados deste estudo sugerem que o nível de desenvolvimento mental se situou abaixo dos valores normativos, o que vai na linha de outros estudos com crianças institucionalizadas. Os trabalhos pioneiros de Provence e Lipton (1962) e de Tizard e Joseph (1970) concluíram que as crianças institucionalizadas apresentam um atraso de desenvolvimento progressivo e estudos mais recentes (Nelson et al., 2007; O'Connor et al., 2000) continuam a apontar para um desempenho intelectual inferior muito significativo. No nosso estudo não se confirmou, contudo, uma associação estatisticamente significativa entre o quociente de desenvolvimento mental e os valores critério de segurança e de dependência. Foi apenas encontrado um resultado marginalmente significativo entre a sub-escala raciocínio prático e a escala proximidade, o que parece indicar que as crianças que manifestam melhor raciocínio prático tendem a apresentar mais comportamentos de aproximação da cuidadora.
No que respeita aos indicadores de psicopatologia, constatou-se que, quer as escalas de internalização, quer as de externalização, se aproximaram dos valores encontrados por Achenbach e Rescorla (2000) para a população clínica. Este achado é congruente com a investigação sobre os efeitos nocivos da privação parental, nas esferas comportamental e social (Hodges \& Tizard, 1989; Johnson et al., 2006). Na verdade, as crianças institucionalizadas têm evidenciado: (a) maior activação e desatenção; (b) dificuldades de comunicação social; (c) comportamento social inadequado e défices de funcionamento interpessoal (Fischer et al., 1997; Kaler \& Freeman, 1994).

Quanto ao comportamento de base segura da criança em interacção com a sua prestadora de cuidados de referência, os valores de segurança encontrados são inconsistentes com os estudiosos que defendem a prevalência de uma organização de apego inseguro (Chisholm, 1998; Hortacsu \& Cesur, 1993; Marcovitch et al., 1997). É de salientar, contudo, que o AQS avalia comportamentos de base segura, o que é distinto da avaliação da organização de (in)segurança com base na situação estranha de Ainsworth, procedimento que tem sido mais usado na investigação. Todavia, os nossos resultados aproximamse dos encontrados em alguns trabalhos científicos que alegam que, pese embora a exposição precoce e prolongada à privação de cuidados familiares, muitas crianças não manifestam distúrbios ao nível do apego (O'Connor et al., 1999). O'Connor et al. (2000) afirmam que a patogenia dos cuidados não é, por si só, capaz de explicar a totalidade deste fenómeno, advertindo que questões como a severidade, a duração e/ou o tipo de privação devem ser igualmente ponderadas. A este propósito, torna-se oportuno notar que o tempo de institucionalização não sugeriu qualquer associação estatisticamente significativa com as variáveis em análise neste trabalho. Outros estudos (Dozier et al., 2001; Howes, 1999) sustentam que a par da adversidade da trajectória desenvolvimental, a qualidade, sensibilidade e responsividade do novo contexto, apoiam a criança no sentido da resolução das suas dificuldades, permitindolhe dirigir os seus comportamentos de apego a uma/s nova/ $\mathrm{s}$ figura/s e reorganizar as suas representações de apego. Consequentemente, e ainda que o presente estudo empírico não tenha incluído o construto de sensibilidade, a meta-análise de Van IJzendoorn, Vereijken, BakermansKranenburg e Risken-Walraven (2004) evidenciou que o AQS se correlaciona fortemente com a sensibilidade. Por conseguinte, a primazia concedida por Ainsworth, Blehar, Waters e Wall (1978) ao construto de sensibilidade materna como elemento-chave para a construção de um apego seguro, parece encontrar aqui alguma expressividade. Todavia, as escalas interacção suave e proximidade de Posada et al. (1995) encontram-se ligeiramente abaixo dos valores registados para a população normativa, o que aponta para alguma desarmonia entre 
a tonalidade emocional da criança e a sua prestadora de cuidados e, nesse sentido, a necessidade de proximidade ser menos proeminente nestas crianças. Importa, no entanto, relevar que o facto do AQS remeter para a observação da criança em interacção com a cuidadora no CAT por um período de tempo considerável, poderá torná-lo menos adequado para avaliar a organização de (in)segurança em contexto institucional (concretamente a escassez de atenção e prestação de cuidadois individualizados), incentivando uma maior disponibilidade da cuidadora, o que associado às necessidades afectivas $\mathrm{e}$ atencionais destas crianças poderá ter concorrido para uma sobre-estimação dos resultados de segurança encontrados.

Verificou-se que a variável idade estava marginalmente relacionada com a escala interacção com outros adultos, o que poderá relacionar-se com as aquisições e tarefas desenvolvimentais próprias do período pré-escolar, na medida em que as novas capacidades representacionais e comunicacionais fazem com que o sistema comportamental de apego se organize em função de internalizações cognitivo-afectivas. A proximidade física à figura de apego deixa de se constituir como o principal objectivo do sistema de apego, dada a capacidade para regular o contacto físico à distância. Por outro lado, a participação em contextos sociais mais alargados, por períodos de tempo mais extensos, potencializa o desenvolvimento de novos relacionamentos que podem vir a constituir-se como dinâmicas de apego importantes (Mayseless, 2005).

Todavia, e apesar das associações entre a idade e a dependência ou interacção com outros adultos terem sido salientadas pela literatura para um percurso de vida normativo, será importante sublinhar que o facto das cuidadoras não despenderem tempo individual diário com as crianças, poderá reflectir níveis de dependência mais baixos que os esperados para a população normativa. Paralelamente, as elevadas necessidades de atenção destas crianças e a presença de um número igualmente elevado de figuras adultas nas instituições, poderão relacionar-se com a associação positiva entre a idade e a escala interacção com outros adultos.

No que respeita às narrativas sobre o apego das prestadoras de cuidados, observou-se que não estavam relacionadas com a segurança do apego das crianças. Estes resultados são inconsistentes com os dados que têm vindo a ser reportados pela literatura (Vaughn et al., 2007; Veríssimo \& Salvaterra, 2006a, 2006b), designadamente com o pressuposto da teoria do apego que remete para o papel mediador dos modelos internos dinâmicos da mãe na organização do comportamento parental e, subsequentemente, na qualidade do apego da criança. Contudo, o nosso estudo apresenta um outro resultado correlação significativa entre representação de apego e nível de escolaridade das cuidadoras - que poderá ajudar a clarificar esta não associação entre a representação das cuidadoras e a segurança da criança.
Para além disto, registaram-se resultados marginalmente significativos entre as histórias mãe-criança e a escala proximidade, o que poderá sugerir abertura da criança para a expressão das suas necessidades, ainda que a cuidadora possa não evidenciar níveis de contacto físico elevados.

Em suma, os dados encontrados parecem ser consistentes com o impacto negativo documentado pela investigação centrada no fenómeno da privação dos cuidados parentais em meio institucional. $\mathrm{Na}$ verdade, além da reprodução dos nefastos resultados nos domínios do desenvolvimento mental e da psicopatologia, os valores próximos do apego seguro revelaram, todavia, especificidades relacionadas com interacções criança-cuidadora menos suaves e harmoniosas pautadas por necessidades de proximidade e de contacto físico menos dominantes. De qualquer modo, convém salientar que esta amostra poderá representar, muito provavelmente, uma população com particularidades tais que demandariam outro conjunto de instrumentos de avaliação, sobretudo no domínio do apego da criança e da representação de apego da prestadora de cuidados de referência.

\section{Considerações Finais}

O presente estudo empírico comporta um conjunto de limitações, que devem ser consideradas. Primeiro, o reduzido número de participantes, que compromete o nível de significância estatística e, portanto, uma compreensão mais robusta e clara dos dados. Segundo, a inexistência de uma linha de avaliação inicial, aquando da entrada da criança na instituição, que dificulta o estudo do impacto da institucionalização, pois face à exposição a múltiplos factores de risco, inerentes ao contexto familiar e institucional, torna-se difícil perceber se os resultados obtidos não poderão também relacionar-se com processos (in)adaptativos precedentes ao momento da colocação institucional. Terceiro, a não aferição das medidas de avaliação do nível de desenvolvimento mental e da sintomatologia psicopatológica, desconhecendo-se pontos de corte normativos nacionais.

Complementarmente, o estudo da relação entre apego e psicopatologia seria enriquecido com a inclusão de uma medida de avaliação da desorganização do apego, uma vez que permitiria identificar e classificar a ausência ou o colapso das estratégias comportamentais requeridas pela criança na interacção com a cuidadora de referência. Por outro lado, dado que a avaliação do apego se baseou apenas na interacção criança-cuidadora, poderia ser útil considerar a resposta da criança a figuras estranhas, ou seja, a extensão deste estudo para o comportamento social indiscriminado. Por fim, com vista a examinar com mais detalhe a qualidade do apego criança-cuidadora, seria pertinente utilizar uma medida de sensibilidade, no sentido de se analisar o 
papel mediador desta variável nos resultados de segurança observados.

Em sentido lato, e apesar dos resultados obtidos sustentarem marginalmente as hipóteses inicialmente colocadas, é importante notar o carácter exploratório deste estudo, que remete para uma abordagem preliminar do impacto da vivência institucional da infância no plano nacional.

\section{Referências}

Achenbach, T. M. (2001). Manual for ASEBA School-Age Forms \& Profiles. Burlington, VT: University of Vermont.

Achenbach, T. M., \& Rescorla, L. A. (2000). Manual for ASEBA Preschool Forms \& Profiles. Burlington, VT: University of Vermont.

Ainsworth, M., Blehar, M., Waters, E., \& Wall, S. (1978). Patterns of attachment: A psychological study of the strange situation. Hillsdale, NJ: Erlbaum.

Bowlby, J. (1973). Attachment and loss: Vol. 2. Separation. Harmondsworth, UK: Penguin Books.

Bretherton, I., \& Munholland, K. A. (1999). Internal working models in attachmet relationships: A construct revisited. In J. Cassidy \& P. R. Shaver (Eds.), Handbook of attachment: Theory, research and clinical implications (pp. 3-20). New York: The Guildford Press.

Castro, S. L., \& Gomes, I. (1996). Adaptação provisória das Escalas Griffiths ao Português. Manuscrito não-publicado, Faculdade de Psicologia e Ciências da Educação, Universidade do Porto, Portugal.

Chisholm, K. (1998). A three year follow-up of attachment and indiscriminate friendliness in children adpted from Romanian orphanages. Child Development, 69, 1090-1104.

Cicchetti, D., \& Tucker, D. (1994). Development and selfregulatory structures of the mind. Development and Psychopathology, 6, 533-549.

Dozier, M., Stovall, K. C., Albus, K. E., \& Bates, B. (2001). Attachment for infants in foster care: The role of the caregiver state of mind. Child Development, 5, 1467-1477.

Fischer, L., Ames, E. W., Chisholm, K., \& Savoie, L. (1997). Problems reported by parents of Romanian orphans adopted to British Columbia. International Journal of Behavioral Development, 20, 67-82.

Frank, D., Klass, P., Earls, F., \& Eisenberg, L. (1996). Infants and young children in orphanages: One view from pedriatics and Child Psychiatry. Pedriatics, 97, 569-578.

Gonçalves, M., Dias, P., \& Machado, B. C. (2007a). Questionário de Comportamentos da Criança 1 1/2 - 5. Manuscrito não-publicado, Universidade do Minho, Braga, Portugal.

Gonçalves, M., Dias, P., \& Machado, B. C. (2007b). Questionário de Comportamentos da Criança 6-18. Manuscrito nãopublicado, Universidade do Minho, Braga, Portugal.

Griffiths, R. (1970). The abilities of young children: A comprehensive system or mental measurement for the first eight years of life. North Dean, UK: The Test Agency.

Gunnar, M., Plorison, S., Chisholm, K., \& Schuder, M. (2001). Salivary cortisol levels in children adopted from Romanian orphanages. Development and Psychopathology, 13, 611628.
Hodges, J., \& Tizard, B. (1989). IQ and behavioural adjustment of ex-institutional adolescents. Journal of Child Psychology \& Psychiatry, 30, 53-75.

Hortacsu, N., \& Cesur, S. (1993). Relationships between depression and attachment styles in parent and institutionreared Turkish children. Journal of Genetic Psychology, 154, 329-337.

Howes, C. (1999). Attachment relationships in the context of multiple caregivers. In J. Cassidy \& P. R. Shaver (Eds.), Handbook of attachment: Theory, research and clinical implications (pp. 671-687). New York: The Guildford Press.

Johnson, R., Browne, K., \& Hamilton-Giachritsis, C. (2006). Young children in institutional care at risk of harm. Trauma, Violence, \& Abuse, 7, 1-26.

Kaler, S. R., \& Freeman, B. J. (1994). An analyses of environmental deprivation: Cognitive and social development in Romanian orphans. Journal of Child Psychology \& Psychiatry, 35, 769-781.

Marcovitch, S., Goldberg, S., Gold, A., Washington, J., Wassom, C., Krekewich, K., et al. (1997). Determinants of behavioural problems in Romanian children adopted in Ontario. International Journal of Behavioural Development, 20, 17-31.

Mayseless, O. (2005). Ontogeny of attachment in middle childhood: Conceptualization of normative changes. In K. A. Kerns \& R. A. Richardson (Eds.), Attachment in middle childhood (pp. 1-23). New York: The Guilford Press.

McCall, R. B., \& Groark, C. J. (2000). The future of applied child development research and public policy. Child Development, 71, 197-204.

Nelson, C. A., Zeanah, C. H., Fox, N. A., Marshall, P. J., Smyke, A. T., \& Guthtrie, D. (2007). Cognitive recovery in socially deprived young children: The Bucharest Early Intervention Project (BEIP). Science, 318, 1937-1940.

O'Connor, T., Bredenkamp, D., Rutter, M., \& The English and Romanian Adoptees Study Team. (1999). Attachment disturbances and disorders in children exposed to early severe deprivation. Infant Mental Health Journal, 20, 10-29.

O’Connor, T. G., Rutter, M., Beckett, C., Keaveney, L., Kreppner, J. M., \& The English and Romanian Adoptees Study Team. (2000). The effects of global severe privation on cognitive competence extension and longitudinal followup. Child Development, 71, 376-390.

Park, K. A., \& Waters, E. (1989). Security of attachment and preschool friendships. Child Development, 60, 10761081.

Posada, G. (2006). Assessing attachment security at age three: Q-Sort home observations and the McArthur Strange Situation Adaptation. Social Development, 15, 644-658.

Posada, G., Waters, E., Crowell, J. A., \& Lay, K. (1995). It is easier to use a secure mother as a secure base? Attachment Q-Sort correlates of Adult Attachment Interview. In E. Waters, B. E. Vaughn, G. Posada, \& K. Kondo-Ikemura (Eds.), Caregiving, cultural, and cognitive perspectives on secure-base behavior and working models: New growing points of attachment theory and research. Monographs of the Society for Research in the Child Development, 60(2/3), 133-145.

Provence, S., \& Lipton, R. C. (1962). Infants in institutions. New York: International Universities Press. 
Smyke, A. T., Koga, S. F., Johnson, D. E., Fox, N. A., Marshall, P. J., Nelson, C. A., et al. (2007). The caregiving context in institution-reared and family-reared infants and toddlers in Romania. Journal of Child Psychology and Psychiatry, 48, 210-218.

Soares, I. (2007). Desenvolvimento da teoria e da investigação da vinculação. In I. Soares (Ed.), Relações de vinculação ao longo do desenvolvimento: Teoria e avaliação (pp. 1345). Braga, Portugal: Psiquilíbrios.

Tizard, B., \& Joseph, A. (1970). Cognitive development of young children in residential care: A study of children aged 24 months. Journal of Child Psychology \& Psychiatry, 11, $117-186$

Tizard, B., \& Rees, J. (1975). The effect of early institutional rearing on the behavioural problems and affectional relationships of four-year-old children. Journal of Child Psychology \& Psychiatry, 61, 61-73.

Van IJzendoorn, M. H., \& Juffer, F. (2006). The Emanuel Miller Memorial Lecture 2006: Adoption as intervention. Metaanalytic evidence for massive catch-up and plasticity in physical, socioemotional and cognitive development. Journal of Child Psychology and Psychiatry, 47, 1228-1245.

Van IJzendoorn, M. H., Vereijken, C. M. J. L., BakermansKranenburg, M. J., \& Risken-Walraven, J. M. (2004). Assessing attachment security with the Attachment Q-Sort: Meta- analytic evidence for the validity of the observer AQS. Child Development, 75, 1188-1213.

Vaughn, B., Coppola, G., Veríssimo, M., Monteiro, L., Santos, A., Posada, G., et al. (2007). The quality of maternal securebase scripts predicts children's secure-base behavior at home in three sociocultural groups. International Journal of Behavioral Development, 31, 65-76.

Veríssimo, M., \& Salvaterra, F. (2006a). Maternal secure base scripts, chidren's attachment security, and mother-child narrative styles. Attachment \& Human Development, 8, 199208.

Veríssimo, M., \& Salvaterra, F. (2006b). O modelo interno dinâmico da mãe e o comportamento de base segura dos seus filhos num grupo de crianças adoptadas. Análise Psicológica, 1(20), 37-50.

Veríssimo, M., \& Santos, A. J. (1999). Instrumentos para a avaliação da vinculação. Manuscrito não-publicado, Instituto Superior de Psicologia Aplicada, Lisboa, Portugal.

Vondra, J., \& Barnett, D. (Eds.). (1999). Atypical attachment in infancy and early childhood among children and developmental risk. Monographs of the Society for Research in Child Development, 64(3).

Waters, E. (1995). The attachment Q-set (Version 3). In E. Waters, B. Vaughn, G. Posada, \& K. Kondo-Ikemura (Eds.), Caregiving, cultural and cognitive perspectives on securebase behaviour and working models: New growing points of attachment theory and research. Monographs of the Society for Research in Child Development, 60(2/3), 234-246. (Original work published 1987)

Waters, H. S., \& Rodrigues-Doolabh, L. (2004). Manual for decoding secure base narratives. Unpublished manuscript, State University of New York at Stony Brook.

Zeanah, C. H., Smyke, A. T., Koga, S. F., Carlson, E., \& The Bucharest Early Intervention Project Core Group. (2005). Attachment in institutionalized and community children in Romania. Child Development, 76, 1015-10. 